\title{
The VA and Non-VA Experience of Tracking Good Care
}

\author{
Erik Langhoff, MD, PhD, ${ }^{1,2}$ Albert Siu, MD, MSPH, ${ }^{1,2}$ Kenneth Boockvar, MD, MS, ${ }^{1-3}$ Linda Bund, MMI, \\ Jim Connell, BS, ${ }^{1}$ and William Hung, MD, MPH ${ }^{1,2}$
}

\begin{abstract}
The VA Mission Act of 2018 allows for choice of health care for 9 million veterans in their community, but deciding where the best care is requires transparency. Recent reports questioning the transparency of reporting health care outcomes in the Department of Veterans Affairs (VA), the largest US health care organization, pointed to flaws in how VA tracks and improves performance, and posed questions about the validity and transparency of using popular hospital ratings systems to define good care. To explore this further, the authors examined 3 widely referenced public health care ranking models - U.S. News America's Best Hospitals, Truven Health Analytics, and Hospital Compare - and the VA model. Upon examination, the authors find that metrics used across the 4 models are neither comparable nor transparent. Between $6 \%-46 \%$ reporting deficiencies were found in reporting of hospital metrics in non-VA hospitals, which reduces transparency for the public. In contrast, VA reporting is $100 \%$. Comparing VA health care and Hospital Compare quality outcomes showed similar or better outcomes for VA for the same metrics of quality and for comparable health care costs. VA inpatient satisfaction falls significantly short of the private sector, but no individual VA outcome measure was found to contribute significantly to inpatient satisfaction. However, overall inpatient satisfaction increased over time with higher global hospital ranking in both VA and non-VA health care. Encouraging use of uniform rating models and reporting of metrics from all hospitals would improve transparency of current health care reporting to the consumer.
\end{abstract}

Keywords: veteran care, quality of care, public reporting

\section{Introduction}

$\mathbf{F}$ OR THE LAST 25 YeARS, quality improvement in health care in the United States has focused on the Triple Aim approach of improving quality, while simultaneously increasing the efficiency of health care delivery, and reducing costs. There is consensus that the quality of health care should be measurable, tracked, and reported but there has been little agreement on how to do that in a transparent way. ${ }^{1,2}$ With passage of the VA Mission Act of 2018, Congress will give 9 million veterans the option to seek care in their local community. A possible shift of market that size affects not only Department of Veterans Affairs (VA) and non-VA hospital operations, but places the veteran at the center in choosing where health care should be provided. Decisions on health care options should be made in an informed and transparent way. Therefore, it is imperative that, when selecting where to receive care, consumers understand the similarities and differences among the ratings systems for hospitals.

Recent articles and reports ${ }^{3-5}$ questioning the transparency of reporting health care outcomes in the VA, the largest US health care organization, pointed to flaws in how VA tracks and improves performance, and posed questions on the validity and transparency of using popular hospital ratings systems to define good care. To explore this further, the research team compares 3 widely referenced public health care ranking models with that of the VA available for veterans seeking alternate care. The goal is to examine the structure of rating models to understand differences in transparency in VA and non-VA outcome measures and to compare common metrics across hospitals stratified by star ratings to understand if star quality ratings are comparable across rating systems. Common metrics between VA and non-VA care quality metrics allow for these comparisons.

\footnotetext{
${ }^{1}$ James J. Peters VA Medical Center, Bronx, New York.

${ }^{2}$ Icahn School of Medicine at Mount Sinai, New York, New York.

${ }^{3}$ The New Jewish Home, New York, New York.
} 
Truven Health Analytics, ${ }^{6}$ America's Best Hospitals, ${ }^{7}$ Hospital Compare, ${ }^{8}$ and Veterans Health Administration (VHA) Strategic Analytics for Improvement and Learning (SAIL), ${ }^{9}$ have emerged as national health care quality frameworks that provide an aggregate assessment of health care quality and at state-level by the Kaiser Family Foundation, ${ }^{10}$ focused mainly on Medicaid policy. SAIL is a tool used to assess VA hospital ratings, and though not all measures are publicly available, SAIL is the basis for the publicly reported measures found on Hospital Compare ${ }^{11}$ for VA Hospitals and the focus of the recent controversial article in the N.Y. Times. ${ }^{3}$ Truven Health Analytics, formerly known as Thomson Reuters (1982), is a multinational company delivering analytic tools, benchmarks, and consulting services to hospitals, government agencies, health plans, and pharmaceutical companies. The VA Office of Informatics and Analytics adopted elements of Truven Health Analytics measures, and added elements to SAIL that are focused on the VA shift to outpatient care, outpatient access, and efficiency. The America's Best Hospital ranking was first published in 1990 by U.S. News and World Report, based on work done by the National Opinion Research Center at the University of Chicago. Hospital Compare was developed in 2003 as an analytics tool for a group of health care organizations - including The Joint Commission (TJC), the Centers for Medicare \& Medicaid Services (CMS), the American Hospital Association, AARP, and other stakeholders in the Hospital Quality Alliance - through which quality data must be submitted to CMS and made available to consumers on the Hospital Compare website. ${ }^{11}$

\section{Methods}

\section{Comparison of rating systems}

The research team used publicly available data to compare and contrast the components of ratings system. The components were organized into hospital structure, patient outcomes (inpatient outcomes and if available outpatient outcomes), and hospital reputation as a reflection of process. The team identified the most commonly used ratings systems and included Hospital Compare, ${ }^{8}$ Truven Analytics, ${ }^{6}$ and, for VA hospitals, VHA SAIL.' ${ }^{9}$ The America's Best Hospital $^{7}$ rating system also was included for comparison because of its popularity and consumer access.

\section{Data collection}

The research team examined the components of the rating systems, which included the following metrics with descriptions as below:

ORYX ${ }^{\circledR}$. TJC integrates outcomes and performance measures into the accreditation process that are publicly reported. ORYX measures are a compilation of preventive, outpatient, inpatient, and surgical performance measures.

Healthcare effectiveness data and information set (HEDIS). The Agency for Healthcare Research \& Quality (ARHQ) developed HEDIS measures as a broad array of outpatient performance measures. HEDIS scores, maintained by the National Committee for Quality Assurance
(NCQA), evaluate and publicly report on the quality of physicians, health maintenance organizations, preferred provider organizations, Accountable Care Organizations, and other organizations. The HEDIS measurement set consists of 83 measures across 5 domains of care with an emphasis on outpatient measures for preventive care (www .ncqa.org/hedis). HEDIS measures are NCQA driven and are widely used performance measures. For adults, the measures address a broad range of outpatient and preventive health factors including cancer screening, diabetes, hypertension, and mental health medication management. HEDIS data also are the centerpiece for "report cards" for most national health plans. ${ }^{12}$

Surgical care improvement project (SCIP). The CMS SCIP is a composite of inpatient core measures for surgical improvement ${ }^{13}$

Hospital consumer assessment of healthcare providers and systems (HCAHPS). HCAHPS is a publicly reported and nationally standardized survey of patients' perspectives and experiences of health care. The HCAHPS survey asks patients 27 questions about their recent hospital experience with 18 core questions about the hospital stay (communication, staff responsiveness, environment, pain management, medication education, discharge education, and overall hospital rating). The survey is administered within 6 weeks after discharge (www.cms.gov).

Medicare provider analysis and review (MEDPAR). The MEDPAR database uses data sets with information on Medicare discharges, the Hospital Compare data set, mortality and 30-day readmission rates, and HCAHPS data (www.cms.gov).

Patient safety index (PSI). The PSI score is based on an AHRQ framework of a PSI composite index but includes only 5 of the 11 components selected, because those have been endorsed by the National Quality Forum, plus 2 additional PSIs, which include deaths deemed to be avoidable.

Hospital-acquired conditions (HAC). In 2005, Congress mandated the HAC initiative, which was implemented in 2008 and was one of the payment reforms implemented by CMS to increase public attention to value-based health care delivery. Under the HAC initiative complications designated "never events" were identified by CMS: Injury from falls, hospital-acquired pressure ulcers, catheter-associated urinary tract infections (CAUTIs), central line-associated bloodstream infections (CLABSIs), air embolism, retained foreign body, deep vein thrombosis or pulmonary embolism, poor glycemic control, blood incompatibility, and surgical site infections.

SAIL. SAIL incorporates 28 metrics including access to care, mortality rates, infection rates, and patient satisfaction. To arrive at outcome scores for SAIL, the VA Office of Informatics and Analytics adopted elements of the Truven Health Analytics measures, but added elements reflecting the VA shift to outpatient care. SAIL assesses VA medical centers across 10 domains -9 domains representing health care quality, and 1 domain for health care efficiency. 
Each of the domains is given a percentage-weighted value of $8 \%-12 \%$ that is used to benchmark VA facilities nationwide. Scores are adjusted for facility complexity and population attributes (VSSC report, VISN [Veterans Integrated Service Network] Support Services Center). SAIL is the basis for publicly reported VA measures and its 5-star rating system and is the basis of the controversy of transparency in the recent article in the New York Times, ${ }^{3}$ which questioned the validity of the star rating in reporting health care outcomes.

\section{Other relevant rating systems}

National Surgical Quality Improvement Program (NSQIP) and VA Surgical Quality Improvement Program (VASQIP): During the late 1980s, the VA came under scrutiny for operative mortality rates in VA hospitals. The perception in Congress was that these rates were above private sector norms. In response, Congress passed a mandate that VA report its surgical outcomes annually, and compare them to national (private sector) averages. Unfortunately, there were no national averages to compare with. However, the VA's extensive information system provided a unique avenue for the development of the $\mathrm{Na}$ tional VA Surgical Risk Study to develop a health outcome model normalized for patient risk factors, effectiveness of care, and random variation based on input from more than 100,000 operations at VA centers. This allowed risk stratification of 30-day mortality and 30-day morbidity in 9 surgical specialties. The success of the model resulted in the development of NSQIP for the entire VA. In 2001 the American College of Surgeons (ACS) became interested in the model's efficiency and the ACS NSQIP was developed and applied for all private sector hospitals. The VA program continues as the VASQIP. ${ }^{14}$

Consumer assessment of healthcare providers and systems (CAHPS). Clinician \& Group Surveys scores are established measures of service quality.

- Getting timely appointments, care, and information (5 items)

- How well providers (or doctors) communicate with patients (6 items)

- Helpful, courteous, and respectful office staff (2 items)

- Patients' rating of the provider (or doctor) (1 item)

\section{Comparison of availability of ratings across VA and non-VA hospitals}

The research team used Hospital Compare data (20122014) and VA SAIL data (2016) to identify availability of metrics within each rating system among all US hospitals. The team identified common metrics among VHA SAIL and CMS Hospital Compare and identified (1) whether data are available in the hospital cohorts (VA vs. non-VA), and (2) what the differences between the rates of quality of care metrics are across VA and non-VA hospitals across star ratings. Hospitals were stratified by star ratings because it is often used and quoted to guide consumer choice.

\section{Statistics}

$t$ Tests were used to determine if mean rates of quality measures are different across groups and coefficient of determination, $R^{2}$, was used to test statistically significant differences and data fit to a statistical model.

\section{Results}

Donabedian was a pioneer in defining quality measures, and divided quality of care into 3 categories: structure, outcome, and process. ${ }^{1,2}$ For each of those categories the research team compared the quality framework of America's Best Hospitals, Truven Health, VHA SAIL, and Hospital Compare.

\section{Structure score}

The structure score (Table 1, top) is based on resource commitment to each of the medical specialties rated, and includes number of discharges, key technologies, nurse staffing, intensivist staffing, recognition as a trauma center and specialized patient service center, and national accreditations (eg, Nurse Magnet Hospital, National Cancer Institute designation). These core elements of the America's Best Hospitals rating are controversial because of the major emphasis (30\% weighting) on hospital structure, including RN Magnet Status, Trauma Center designation, and offering of medical specialties, which is related solely to inpatient services and is unlikely to reflect the quality of outpatient care delivery. Furthermore, as currently calculated, hospital structure score does not include investment in electronic health records (EHRs), information technology services, quality assurance, and patient safety. Truven Health Analytics, VHA SAIL, and Hospital Compare do not include those elements but VHA SAIL includes RN turnover rate.

\section{Outcomes score}

Outcomes score is defined by the health status of patients and populations resulting from care. Table 1 (middle) shows a comparison of the 4 quality frameworks. Inpatient outcome measures including hospital mortality and morbidity are incorporated into all schemes. Length of stay, 30-day readmission rate (considered a consequence of inpatient care, failure to provide outpatient follow-up, and variation between systems), and admission for ambulatory caresensitive conditions (considered a consequence of outpatient care, failure to proactively monitor chronic conditions such as congestive heart failure $[\mathrm{CHF}]$ and diabetes) are included in VHA SAIL but not consistently in the other frameworks, and VHA SAIL weights outpatient quality measures to a greater extent. The Hospital Compare website and VHA SAIL both use a star system to rank hospitals from 1 to 5 ( 1 being lowest and 5 being highest). Publicly available data sets were obtained for both sets of facilities from their respective websites. There were 146 facilities included in the fiscal year 2016 Quarter 1 SAIL scorecard reports. There were 3544 facilities included in the Hospital Compare database as of June 2016. Even though many more facilities were included in the Hospital Compare database, the distribution of overall star rating (Figure 1) and regional star rating (data not shown) were comparable and not suggestive of inconsistencies between SAIL and Hospital Compare. 


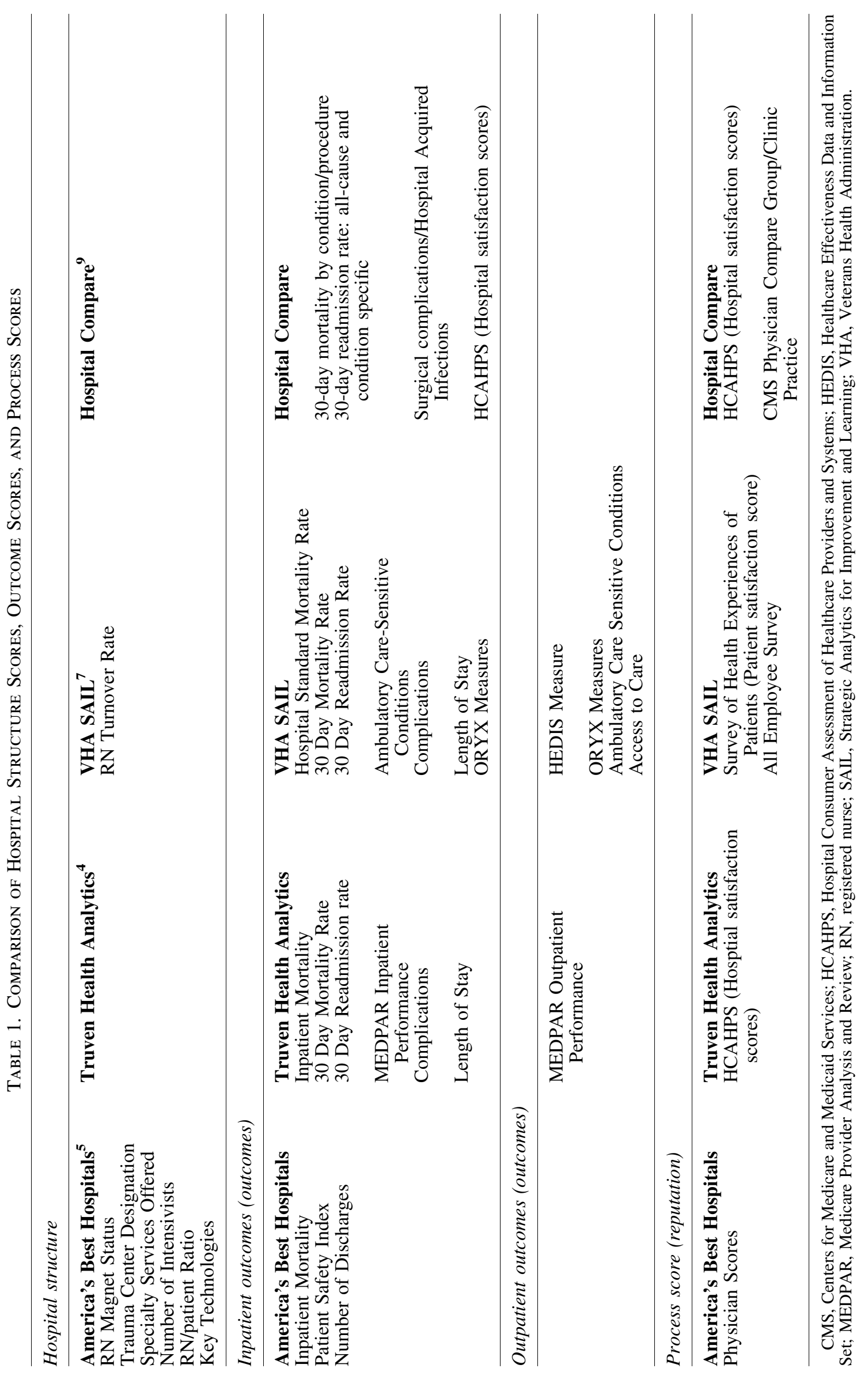




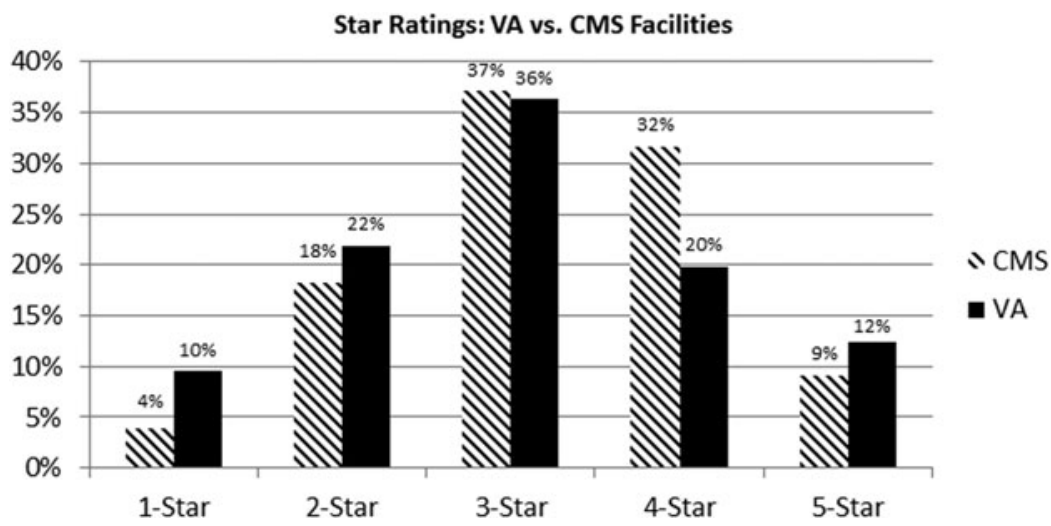

FIG. 1. Distribution of hospital star ratings, VA and Medicare-certified hospitals included in publicly available data sets as of June 2016. VA, US Department of Veterans Affairs.

A comparison of quality measures between VA hospitals and Medicare-certified hospitals from the Hospital Compare website was performed. Not all reported measures were comparable between the 2 health care hospital systems but many were. Table 2 shows a comparison of mean scores for quality measures between VA and Medicare-certified hospitals included in the data sets. Means were compared for statistical significance using 2-sample $t$ tests assuming unequal variances. For each of the quality measures VA hospitals scored better than non-VA hospitals on Hospital Compare. However, for patient satisfaction measured using HCAHPS, the VA experience falls short of non-VA hospitals on Hospital Compare.

The Hospital Compare data included many non-VA facilities that did not report on several quality measures $-6 \%$ to $46 \%$ of facilities, depending on the measure - whereas all VA Hospitals had data for all measures. In addition, 1099 hospitals on Hospital Compare were completely excluded from analysis because of the sparseness of their data. These data show that there are a significant number of non-VA hospitals without public reporting primarily because of the size of these hospitals whereas VA hospitals have public reporting regardless of the size of the hospitals; this in turn may allow consumers access to these data. As an integrated health care system with inpatient and outpatient components that serves a population, VA hospitals have additional metrics on access to care that do not have a counterpart in non-VA health care.

\section{Reputation}

A reputation (patient satisfaction scores) comparison is shown in Table 1. Reputation between Truven Health Analytics, VHA SAIL, and Hospital Compare are measured by inpatient satisfaction (using the Survey of Health Experiences of Patients or HCAHPS). Outpatient measures are not included in America's Best Hospitals or Hospital Compare. Very differently, America's Best Hospitals does not ascertain inpatient or outpatient satisfaction. Inpatient satisfaction with the VA experience falls short of the nonVA hospitals on Hospital Compare (Table 2).

The research team first examined whether trends or differences in resources invested in health care would be a factor of influence. The overall percent health care spending

Table 2. Comparison of Quality Measures Between VA Hospitals* and Non-VA Hospitals FROM THE HOSPITAL COMPARE Website**

\begin{tabular}{|c|c|c|c|c|c|c|}
\hline \multicolumn{3}{|l|}{$V A S A I L$} & \multicolumn{3}{|c|}{ CMS hospital compare } & \multirow{2}{*}{$\frac{t \text { test }}{\mathrm{P}}$} \\
\hline Measure & $N$ & Mean & $N$ & Mean & Not reporting & \\
\hline Acute MI Mortality Rate & 146 & 9.27 & 2394 & 14.166 & 1150 & $<0.0001$ \\
\hline CHF Mortality Rate & 146 & 7.79 & 3254 & 11.69 & 290 & $<0.0001$ \\
\hline Pneumonia Mortality Rate & 146 & 8.67 & 3308 & 11.54 & 236 & $<0.0001$ \\
\hline Catheter-Associated Urinary Tract Infections & 146 & 0.98 & 2488 & 7.83 & 1056 & $<0.0001$ \\
\hline Central Line-Associated Bloodstream Infections & 146 & 0.806 & 2036 & 8.14 & 1508 & $<0.0001$ \\
\hline Methicillin-Resistant Staphylococcus aureus Infections & 146 & 0.10 & 1889 & 4.91 & 1655 & $<0.0001$ \\
\hline 30-day All-Cause Readmission Rate & 146 & 12.17 & 3544 & 15.22 & 0 & $<0.0001$ \\
\hline Acute MI 30-day Readmission Rate & 146 & 15.59 & 2199 & 17.00 & 1345 & $<0.0001$ \\
\hline CHF 30-day Readmission Rate & 146 & 19.39 & 3279 & 22.02 & 265 & $<0.0001$ \\
\hline Pneumonia 30-day Readmission Rate & 146 & 14.76 & 3321 & 16.99 & 223 & $<0.0001$ \\
\hline Inpatient Satisfaction & 146 & 65.13 & 3544 & 88.59 & 0 & $<0.0001$ \\
\hline
\end{tabular}

*VA hospital data from SAIL scorecards fiscal year 2016, quarter 1 (Oct-Dec 2015).

**Hospital compare data from 2012-2014 - the latest available as of June 10, 2016.

CMS, Centers for Medicare \& Medicaid Services; CHF, congestive heart failure; MI, myocardial infarction; SAIL, Strategic Analytics for Improvement and Learning; VA, US Department of Veterans Affairs. 
per capita increased in VA and non-VA health care from 2009 to 2014 by $11.4 \%$ and $8.5 \%$, respectively, when the VA experienced the greatest increase in the number of enrolled veterans (data not shown). The increased spending in health care (VA versus non VA) has not brought proportional dividends of increased patient satisfaction within either health care system (Figure 2).

Because the level of health care funding in the private and the VA system has been stable and comparable over time, and has not changed the perception of inpatient satisfaction in either system, the research team concluded that lower inpatient experience ratings in the VA system were not a result of disparate resource allocations affecting quality metrics. The team next attempted to correlate star quality ratings with inpatient experience. Table 3 shows the same quality measures for VA and non-VA hospitals on Hospital Compare by star rating. Cells highlighted in gray represent facilities that have a lower (ie, better) mean value for a measure than 5-star facilities do. This means that facilities with a higher star rating might not have better quality in all areas measured. Non-VA hospitals on Hospital Compare with a 1-star rating did better on 4 of 11 measures than 5star hospitals. In VA hospitals this was the case for only 1 measure. These results show that hospitals' overall star ranking can give a false perception of level of quality of care across all of the individual measures.

Furthermore, when examining the correlation coefficients $\left(R^{2}\right)$ for each of 10 quality measures (acute myocardial infarction $[\mathrm{MI}]$ mortality rate, $\mathrm{CHF}$ mortality rate, pneumonia mortality rate, CAUTIs, CLABSIs, methicillin-resistant Staphylococcu aureus infections, 30-day all-cause readmission rate, acute MI 30-day readmission rate, $\mathrm{CHF} 30$-day readmission rate, and pneumonia 30-day readmission rate), and patient satisfaction by hospital type, no single quality measure, of the 10 examined, was correlated with patient satisfaction $\left(R^{2}\right.$ : 0.002-0.09). However, when all measures were compounded into a single star quality score (Figure 3 ) the $R^{2}$ for VA hospitals is 0.22 , while the $R^{2}$ for non-VA hospitals is 0.89 , which indicates a strong correlation.

\section{Discussion}

The VA Mission Act of $2018^{15}$ allows for choice of health care for 9 million veterans; they may seek care outside the VA if travel time to the nearest VA is greater than 30 minutes. The extent to which veterans will seek non-VA care remains to be determined. Although the relationship between quality rating and consumer choice of where they receive care is not well established, the change in VA policy on reimbursement for community care to allow for enhanced choice for VA and non-VA care may make the comparison between VA and non-VA care more relevant than before. The transparency of how hospital quality rating systems are composed and communicated may influence veterans' choice of where to receive care based on access to information on hospital structure, hospital outcome, and satisfaction with care. This likely will have significant financial impact on VA health care as well as non-VA health care systems. VA is now providing outcome data directly to CMS, giving users direct and transparent access to compare between VA and non-VA hospitals' reporting for very similar metrics of outcomes and satisfaction. Truven Health Analytics shares many similarities with Hospital Compare and VHA SAIL but U.S. News America's Best Hospitals has very few similarities, and therefore it is untenable for comparison purposes. Present results are in agreement with those of 2 recent studies ${ }^{16,17}$ that quality of care provided by VA is comparable to, or better than, that of non-VA community hospitals. A study by the RAND Corporation showed that in a tally of 83 different measures covering a variety of types of care including safety and effectiveness of treatment, the quality of VA health care exceeded that of non-VA care. ${ }^{18}$

However, present study results suggest that there is between $6 \%-46 \%$ variability in non-VA hospital public reporting of outcomes by Hospital Compare compared to $100 \%$ VA reporting. Although the variability in reporting is likely related to the smaller size of hospitals without public reporting, VA reports data on its hospitals regardless of size;

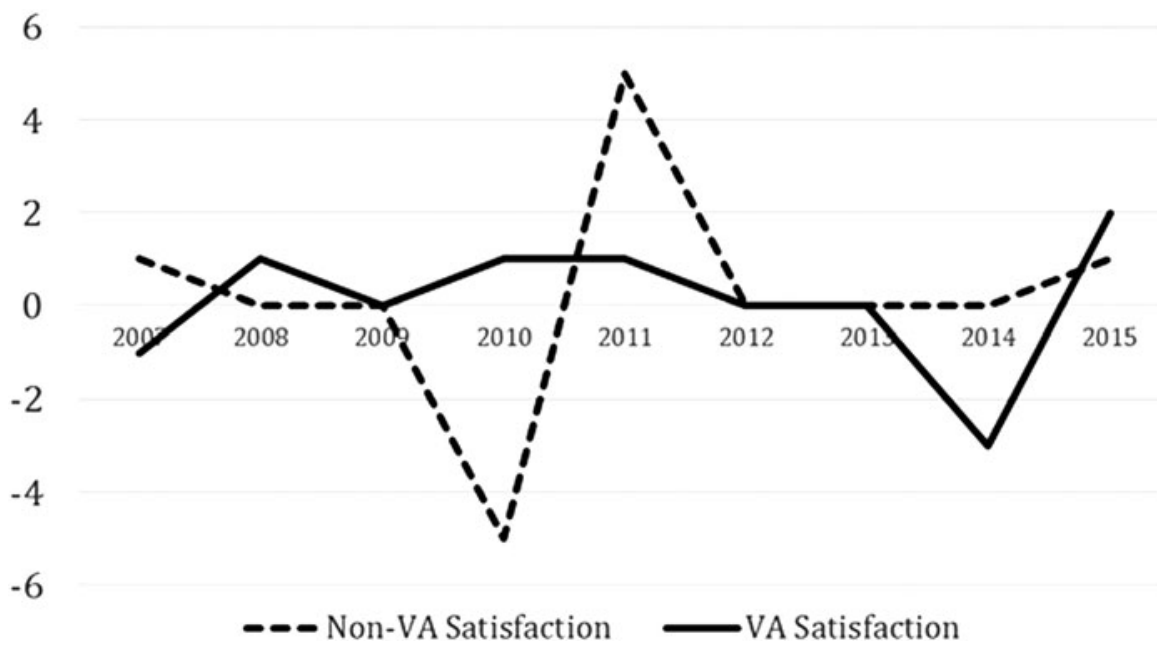

FIG. 2. Change in patient satisfaction for hospital inpatients since 2006 - VA and non-VA (point change on scale of 1100). VHA inpatient satisfaction survey data use until 2012. From 2012 VHA changes to the Hospital Consumer Assessment of Healthcare Providers and Sysytems survey. Data prior to 2012 are not publicly available. VA, US Department of Veterans Affairs; VHA, Veterans Health Administration. 
there are markets, mainly rural, in which a VA hospital with publicly reported data has no non-VA counterpart in Hospital Compare. ${ }^{19}$ This may negatively affect consumers' ability to compare hospitals in their region across VA and non-VA health systems. The VA Mission Act requires the VA Secretary to establish a system or systems for monitoring the quality of care provided to covered veterans through a network assessing the quality of hospital care, medical services, and extended care services, and standards for access to care. The mechanisms to fulfill these requirements remain to be determined because EHRs are not shared between the VA and community hospitals. Complete and transparent reporting of outcome results to Hospital Compare would be key to begin to fulfill these mandates. Of note, the VA, being an integrated health care system for a population of veterans with inpatient and outpatient components, has used metrics for both inpatient and outpatient care; despite some non-VA hospitals having outpatient clinics, metrics on outpatient care are not similarly available for public review.

In 2014, the VA created a patient experience department to ensure consistent measures of patient experience and patient satisfaction across the system. Patient experience measures are direct and personal, whereas the more technical composites of standard quality metrics guide health care systems in monitoring process and outcomes. One of the biggest challenges for health care going forward is determining how to effectively communicate patient satisfaction and experience to patients in a way that helps them select the best care. The HCAHPS survey - which assesses patients' perspectives and experiences of health care including communication, staff responsiveness, environment, pain management, medication education, discharge instructions, and overall hospital rating - was adopted by the VA in 2015. Several recent studies support that higher patient satisfaction is associated with lower 30-day readmission rates, improved adherence to clinical guidelines, and lower inpatient mortality rates, suggesting that patients are good discriminators of the quality of care they receive. ${ }^{20}$ However, not all categories of patients' experiences assessed in the HCAHPS survey contribute equally to overall patient satisfaction. Quality of communication with nurses has the strongest correlation with overall inpatient satisfaction whereas communication with physicians and environment (eg, noise, room cleanliness) were of lower ranking. ${ }^{20}$ America's Best Hospitals does not include inpatient or outpatient satisfaction, but ascertains hospital reputation through a peer review rating submitted by 200 randomly selected, board-certified physicians from across the country; it is based on their responses for each of 16 specialties, collected on a 1-page single-sided questionnaire. Respondents select up to 5 hospitals in their specialty that provide care for serious conditions, without regard to location or cost. The process (reputation) score of America's Best Hospitals carries a significant weight of $25 \%$ of the total score. Its ratings do not account for patient experience or consumer perception of quality.

The lower inpatient satisfaction with VA health care vs. non-VA health care is unexplained given the fact that this study finds that VA and non-VA health care systems have developed parallel systems and are using comparable metrics of cost and quality. The discrepancy between satisfaction and 
VA SAIL

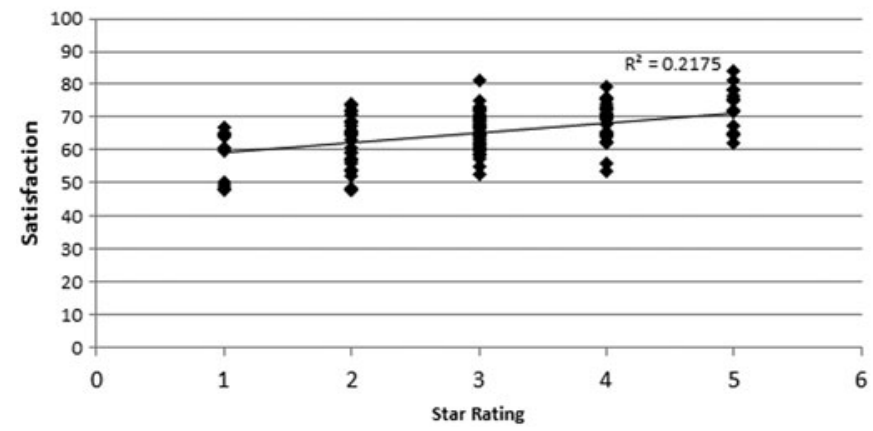

CMS Compare

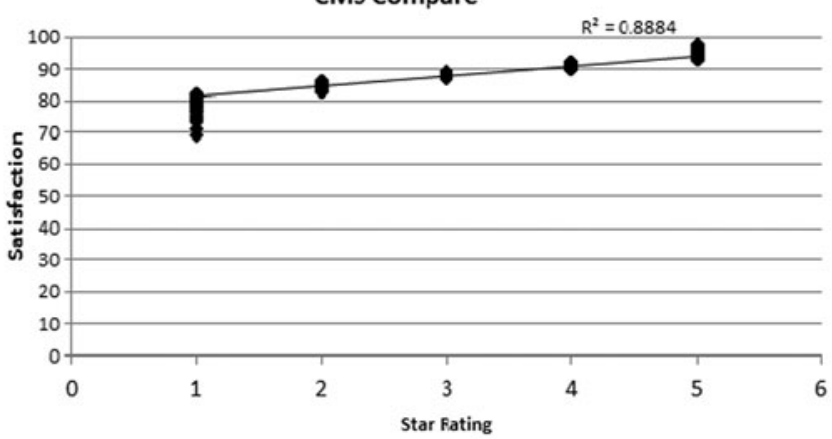

FIG. 3. Correlation between star rating and patient satisfaction: VA and non-VA hospitals. SAIL, Strategic Analytics for Improvement and Learning; VA, US Department of Veterans Affairs.

quality of outcomes could be related to prior studies on the association of quality and satisfaction being conducted in non-VA settings; these may not be applicable to comparisons across VA and non-VA systems, particularly given differences in patient population, cultural differences in a unique population (veterans), and perhaps expectations, and also differences in how the integrated VA health system is established with its inpatient, outpatient, pharmacy, and other benefit programs under one system. Although overall patient satisfaction improves in both health care systems with better overall quality measures, there is a gap in perception of quality.

The N.Y. Times article ${ }^{3}$ emphasizes the need to further understand patients' perceptions of good care vis-a-vis prescribed standardized matrices of quality care metrics defined in hospital ranking models. Blay et $\mathrm{al}^{16}$ also reported lower VA patient satisfaction, but the study does not account for the variability of reporting in Hospital Compare. Because data are reported at the facility level, there is no way to know if patients responding to the satisfaction survey were the same ones being counted in the quality measures. In the case of mortality rates, they certainly were not, as satisfaction surveys are generally given at the end of an inpatient stay, or mailed to the patient's home after discharge. It also is possible that services not directly related to patient care, such as transportation and pharmacy benefits provided by VA care but not by non-VA hospitals, may influence VA overall patient satisfaction. Such elements could be degree of service connection, pharmacy, and transportation benefits that are special to the VA health care system. There are other elements of differences between VA and non-VA care that have not been discussed in this study that potentially could influence patient satisfaction, such as differences between the 2 populations in terms of age, sex, and complexity of disease burden. The median age of the general population is 44 years vs. 64 years median age of all veterans. The age difference may set different expectations of experience (https://www.va.gov/vetdata/docs/Special Reports/KeyStats.pdf).

Likewise, the aging infrastructure of many VA hospitals is part of the patient experience. These are all possible nondirect care-related contributors to the veterans' patient experience that need to be further investigated. VA health care is an integrated system providing eligibility for pharmacy benefits, patient travel benefits, and home care. Such addi- tional elements also can affect veterans' expectations. The lower $R^{2}$ correlation between VA patient satisfaction and VA star quality score (Figure 3) may support that such elements external to direct care contribute to the care experience. In addition, the recent years of controversy on VA care, ${ }^{3}$ forming negative contagions in public media, ${ }^{21}$ also may contribute to lower VA patient satisfaction. These are all elements to be explored further. Ultimately, while the overall quality of VA care is recently reported comparable to or better than non-VA care, the patient's perception of what is good care is most likely a key driver for future health care choices.

In that sense, the lower inpatient satisfaction at present may be a disadvantage to the VA as the Mission Act allows for choice of health care for veterans in the community. A key expectation of the Mission Act is provision of timely access to health care in the VA but also in the community. This sets the expectations for veterans to have that information to make decisions on health care choices. The VA measures timely access to outpatient care but that is not part of the Hospital Compare metrics. Going forward, both VA and non-VA health care systems likely will continue to keep costs low, along with pressure from patients and advocate groups to keep improving transparency and quality of care. To that end, it is important for VA and non-VA systems to align their multidimensional quality measurements to ensure that the transformation sustains transparency and meets the expectations of customers' experience of good care. In health care and other industries, the road map to customer loyalty and sustained success are likely the same.

\section{Acknowledgments}

We thank Dr. T. Fojo, Columbia Presbyterian Hospital and James J. Peter's VAMC, and Dr. M. Sano, James J. Peter's VAMC for review and discussion of the manuscript, and Dr. W.A. Haseltine, ACCESS Health International Inc., for discussion of the manuscript.

\section{Author Disclosure Statement}

The authors declare that there are no conflicts of interest. This work was supported with resources and the use of facilities at the James J. Peters VA Medical Center, Bronx, NY. The contents do not represent the views of the US Department of Veterans Affairs or the US Government. 
Dr. Hung receives support from the Agency for Healthcare Research and Quality Grant 1K08HS022924-01A1.

\section{References}

1. Donabedian A. The quality of care. How can it be assessed? JAMA1988;260:1743-1748.

2. Donabedian A. Evaluating the quality of medical care. Millbank Q 2005;83:691-729.

3. Philipps D. Cancelled Operations. Unsterile Tools. The V.A. Gave This Hospital 5 Stars. 2018. https://www.nytimes.com/ 2018/11/01/us/veterans-hospitals-rating-system-star.html. Accessed April 9, 2019.

4. US Government Accountability Office. VA Health Care Quality: VA Should Improve the Information It Publicly Reports on the Quality of Care at Its Medical Facilities. 2017. https://www.gao.gov/products/GAO-17-741 Accessed April 9, 2019.

5. Slack D, Sallah M. VA conceals shoddy care and health workers' mistakes. 2017. https://www.usatoday.com/ story/news/2017/10/11/va-conceals-shoddy-care-and-healthworkers-mistakes/739852001/ Accessed April 9, 2019.

6. Truven Health Analytics. 15 Top Health Systems Study. 2015. http://truvenhealth.com/Portals/0/assets/0417_15_ Top_Health_Systems_Study.pdf Accessed May 5, 2019.

7. McFarlane E, Murphy J, Olmsted MG, Drozd EM, Hill C. 2009 Methodology: “America's Best Hospitals." 2009. https://www.rti.org/sites/default/files/resources/abhmethod_ 2009.pdf Accessed May 7, 2019.

8. Hospital Compare. Centers for Medicare \& Medicaid Services. https://www.cms.gov/medicare/quality-initiativespatient-assessment-instruments/hospitalqualityinits/hospital compare.html Accessed May 5, 2019.

9. Strategic Analytics for Improvement and Learning (SAIL). https://www.va.gov/qualityofcare/measure-up/strategic analytics_for_improvement_and_learning_sail.asp Accessed May 5, 2019.

10. State Health Facts. Kaiser Family Foundation. http://kff .org/statedata Accessed May 5, 2019.

11. Hospital Compare. Medicare.gov. The Official U.S. Government site for Medicare. https://www.medicare.gov/hospital compare Accessed May 5, 2019.
12. HEDIS and Performance measurement. https://www.ncqa .org/hedis/ Accessed May 5, 2019.

13. Surgical Care Improvement Project (SCIP). Specifications Manual for Joint Commission National Quality Core Measures. 2010. https://manual.jointcommission.org/releases/ archive/TJC2010B/SurgicalCareImprovementProject.html. Accessed May 5, 2019.

14. Fuchshuber PR, Greif W, Tidwel CR, Klemm MS, Frydel C, Wali A. The power of the National Surgical Quality Improvement Program-achieving a zero pneumonia rate in general surgery patients. Permanente J 2012;16:39-45.

15. McCain JS, Akara DK, Johnson SR. VA mission act of 2018. Washington DC: United States Congress, 2018.

16. Blay E Jr, DeLancey JO, Hewitt DB, Chung JW, Bilimoria KY. Initial public reporting of quality at Veterans Affairs vs non-Veterans Affairs hospitals. JAMA Intern Med 2017; 177:882-885.

17. Tanielian T, Farmer CM, Burns RM, Duffy EL, Setodji CM. Are private health care providers ready to treat veterans? Santa Monica, CA: RAND Corporation, 2018.

18. Farmer CM, Hosek SD, Adamson DM. Balancing demand and supply for Veterans' Health Care. Santa Monica, CA: RAND Corporation, 2016.

19. Weeks WB, West AN. Veterans Health Administration hospitals outperform non-Veterans Health Administration hospitals in most health care markets. Ann Intern Med 2018. [Epub ahead of print]; DOI: 10.7326/M18-1540.

20. Manary MP, Boulding W, Staelin R, Glickman SW. The patient experience and health outcomes. N Engl J Med 2013;368:201-203.

21. Kramer AD, Guillory JE, Hancock JT. Experimental evidence of massive-scale emotional contagion through social networks. Proc Natl Acad Sci U S A 2014;111:8788-8790.

Address correspondence to: Erik Langhoff, $M D, P h D$

James J. Peters VA Medical Center 130 West Kingsbridge Road Bronx, NY 10468

E-mail: erik.langhoff@va.gov 